\title{
Irreducible Markov systems on Polish spaces
}

\author{
by \\ Katarzyna Horbacz and Tomasz Szarek (Katowice)
}

\begin{abstract}
Contractive Markov systems on Polish spaces which arise from graph directed constructions of iterated function systems with place dependent probabilities are considered. It is shown that their stability may be studied using the concentrating methods developed by the second author [Dissert. Math. 415 (2003)]. In this way Werner's results obtained in a locally compact case [J. London Math. Soc. 71 (2005)] are extended to a noncompact setting.
\end{abstract}

1. Introduction and notation. In [1] Barnsley et al. considered the evolution of distributions due to the action of randomly chosen transformations - so-called iterated function systems with complete connections. In their paper sufficient conditions for the existence of an invariant measure and stability are formulated.

In [7] A. Lasota and J. Yorke generalized the results mentioned above. The novelty of their study consists in the use of the lower bound technique developed earlier for Markov operators acting on $L^{1}$ spaces.

Recently Werner $[13,14]$ has extended iterated function systems with complete connections to a more general setting. Indeed, he has studied graph directed constructions on locally compact spaces with an open partition (see also [8]).

Barnsley et al. and Werner tackled the problem of the existence of an invariant measure from the probabilistic point of view. In this paper we aim to show that Werner's result may be obtained by functional analysis methods even in the general case of complete separable metric spaces. In fact, Werner's assumption looks somewhat restrictive. For instance it rules out a lot of processes defined on function spaces. Such spaces appear not only when one studies Markov systems generated by partial differential equations (see for instance [6]) but also in random processes describing various phenomena in physics and biology (see for instance [5]). Although there are a huge

2000 Mathematics Subject Classification: Primary 60J10; Secondary 28A80.

Key words and phrases: contractive Markov systems, iterated function systems, invariant measures. 
number of results on Markov processes associated with random iteration of maps (see $[2-4,9-11]$ ), none of our results is covered by the existing theory.

Let $(K, d)$ be a complete and separable metric space. Assume that $K=$ $\bigcup_{j=1}^{N} K_{j}$, where $K_{1}, \ldots, K_{N}$ are such that inf $\left\{d(x, y): x \in K_{m}, y \in K_{n}\right\}>0$ for $m \neq n$. We denote by $\mathcal{L}^{0}(K)$ the space of all bounded Borel measurable functions $f: K \rightarrow \mathbb{R}$, and by $C(K)$ the subspace of all bounded continuous functions. These spaces are equipped with the supremum norm. Moreover, $P(K)$ denotes the space of all Borel probability measures on $K$.

For each $j \in\{1, \ldots, N\}$, let

$$
w_{j 1}, \ldots, w_{j L_{j}}: K_{j} \rightarrow K
$$

be a family of Borel measurable maps such that for each $m \in\left\{1, \ldots, L_{j}\right\}$, there exists $n \in\{1, \ldots, N\}$ such that $w_{j m}\left(K_{j}\right) \subset K_{n}$. Furthermore, for each $j \in\{1, \ldots, N\}$, let

$$
p_{j 1}, \ldots, p_{j L_{j}}: K_{j} \rightarrow[0,1]
$$

be a family of Borel measurable probability functions, that is, $p_{j m}>0$ for all $j, m$ and $\sum_{m=1}^{L_{j}} p_{j m}(x)=1$ for all $x \in K_{j}$.

Following Werner (see [11]) we call $V=\{1, \ldots, N\}$ the set of vertices, and the subsets $K_{1}, \ldots, K_{N}$ the vertex sets. Further, we call

$$
E=\left\{\left(j, n_{j}\right): j \in\{1, \ldots, N\}, n_{j} \in\left\{1, \ldots, L_{j}\right\}\right\}
$$

the set of edges and we write

$$
p_{e}:=p_{j n} \quad \text { and } \quad w_{e}:=w_{j n} \quad \text { for } e=(j, n) \in E .
$$

For an edge $e \in E$ we denote by $i(e)$ the initial vertex of $e$, that is, $i(e)=j$ if and only if $e=(j, k)$ for some $k \in\left\{1, \ldots, L_{j}\right\}$. The terminal vertex $t(e)$ for $e=(j, n) \in E$ is equal to $k$ if and only if $w_{e}\left(K_{j}\right) \subset K_{k}$.

We recall that the quadruple $G=(V, E, i, t)$ is called a directed multigraph or digraph. A sequence (finite or infinite) $\left(\ldots, e_{-1}, e_{0}, e_{1}, \ldots\right)$ of edges is called a path if $t\left(e_{k}\right)=i\left(e_{k+1}\right)$ for all $k$.

For every $j \in V$ we denote by $l(j)$ the smallest number, say $k$, such that there is a path $\left(e_{1}, \ldots, e_{k}\right)$ with $i\left(e_{1}\right)=j$ and $t\left(e_{k}\right)=j$.

A path $\mathbf{c}=\left(e_{1}, \ldots, e_{m}\right)$ is called a cycle if $i\left(e_{1}\right)=t\left(e_{m}\right)$. A cycle is called simple if it does not contain any other cycle. Let $l(\mathbf{c})$ denote the length of $\mathbf{c}$, i.e., $l(\mathbf{c})=m$ if $\mathbf{c}=\left(e_{1}, \ldots, e_{m}\right)$.

We denote by $\left[k_{1}, \ldots, k_{M}\right]$ the greatest common divisor of $k_{1}, \ldots, k_{M} \in \mathbb{N}$.

We call the family $\left(K_{i(e)}, w_{e}, p_{e}\right)_{e \in E}$ a Markov system. A Markov system is irreducible if and only if its directed graph is irreducible, that is, there is a path from any vertex to any other. An irreducible Markov system is said to have period $p$ if the set of vertices can be partioned into $p$ nonempty subsets $\Omega_{1}, \ldots, \Omega_{p}$ such that

$$
i(e) \in \Omega_{j} \Rightarrow t(e) \in \Omega_{j+1} \bmod p
$$


for all $e \in E$, and $p$ is the largest number with this property. An irreducible Markov system with period 1 is called aperiodic.

Finally, $\mathbf{C}=\left\{\mathbf{c}_{1}, \ldots, \mathbf{c}_{M}\right\}$ denotes the set of all simple cycles in $\left(K_{i(e)}, w_{e}, p_{e}\right)_{e \in E}$.

REMARK 1. If an irreducible Markov system $\left(K_{i(e)}, w_{e}, p_{e}\right)_{e \in E}$ is aperiodic, then

$$
\left[l\left(\mathbf{c}_{1}\right), \ldots, l\left(\mathbf{c}_{M}\right)\right]=1 .
$$

Indeed, assume contrary to our claim that $\left[l\left(\mathbf{c}_{1}\right), \ldots, l\left(\mathbf{c}_{M}\right)\right]>1$. Let $p>1$ be a prime number dividing each $l\left(\mathbf{c}_{j}\right)$. By a simple induction on the number of cycles we then show that the system is periodic with period $p$.

To define a Markov operator on $\mathcal{L}^{0}(K)$ associated with the Markov system under consideration we extend $p_{j m}$ onto the whole space $K$ by zero; the maps $w_{j m}$ are extended arbitrarily.

We define a Markov operator on $\mathcal{L}^{0}(K)$ by

$$
U f:=\sum_{e \in E} p_{e} f \circ w_{e} \quad \text { for all } f \in \mathcal{L}^{0}(K)
$$

and its adjoint operator on $P(K)$ by

$$
\int_{K} f d U^{*} \nu=\int_{K} U f d \nu \quad \text { for all } f \in \mathcal{L}^{0}(K) \text { and } \nu \in P(K) .
$$

REMARK 2. Observe that by the Lebesgue monotone convergence theorem, $U$ may be extended to all nonnegative Borel measurable functions (not necessarily bounded).

For $K$ unbounded, a continuous function $L: K \rightarrow[0, \infty)$ is called a Lyapunov function if

$$
\lim _{\varrho\left(x, x_{0}\right) \rightarrow \infty} L(x)=\infty
$$

for some $x_{0} \in X$.

An irreducible Markov system $\left(K_{i(e)}, w_{e}, p_{e}\right)_{e \in E}$ is called weakly asymptotically stable if there exists an invariant measure $\mu_{0} \in P(K)$, i.e., $U^{*} \mu_{0}=$ $\mu_{0}$ and

$$
\lim _{n \rightarrow \infty} \int_{K} U^{n} f(x) \mu(d x)=\int_{K} f(x) \mu_{0}(d x)
$$

for all $f \in C(K)$ and $\mu \in P(K)$.

For $\mu \in P(K)$ we denote by $\omega(\mu)$ the set of all weak limits of subsequences of $\left(U^{* n} \mu\right)_{n \geq 1}$.

We will assume that the Markov system $\left(K_{i(e)}, w_{e}, p_{e}\right)$ is contractive, i.e., there exists $0<a<1$ such that

$$
\sum_{e \in E} p_{e}(x) d\left(w_{e}(x), w_{e}(y)\right) \leq a d(x, y)
$$


for all $x, y \in K_{j}$ and $j \in\{1, \ldots, N\}$. Finally, recall that a function $f$ : $(K, d) \rightarrow \mathbb{R}$ is called Dini continuous if there is $c>0$ such that

$$
\int_{0}^{c} \frac{\Phi(t)}{t} d t<\infty
$$

where $\Phi$ is the modulus of uniform continuity of $f$, that is,

$$
\Phi(t)=\sup \{|f(x)-f(y)|: d(x, y) \leq t, x, y \in K\} .
$$

Our main results concerning irreducible Markov systems defined on Polish spaces are the following:

TheOREM 1.1. Let $\left(K_{i(e)}, w_{e}, p_{e}\right)_{e \in E}$ be a contractive Markov system such that every $p_{e}$ is Dini continuous on $K_{i(e)}$. Then the system has an invariant measure $\mu_{0} \in P(K)$. Moreover the set $\omega(\mu)$ is nonempty for each $\mu \in P(K)$ and the set $\bigcup_{\mu \in P(K)} \omega(\mu)$ is tight.

TheOREM 1.2. Let $\left(K_{i(e)}, w_{e}, p_{e}\right)_{e \in E}$ be an irreducible contractive Markov system such that $\left.p_{e}\right|_{K_{i(e)}}$ is Dini continuous and there exists $\delta>0$ such that $\left.p_{e}\right|_{K_{i(e)}} \geq \delta$ for all $e \in E$. If, in addition, the system is aperiodic, then it is weakly asymptotically stable.

2. Auxiliary lemmas. We start with two definitions. An irreducible Markov system $\left(K_{i(e)}, w_{e}, p_{e}\right)_{e \in E}$ is called globally concentrating if for every $\varepsilon>0$ and every bounded Borel set $A \subset K$ there exist a bounded Borel set $B \subset K$ and $n_{0} \in \mathbb{N}$ such that

$$
U^{* n} \mu(B) \geq 1-\varepsilon \quad \text { for } n \geq n_{0}, \mu \in P(A),
$$

where $U^{*}$ is given by (1.2).

The following lemma gives a condition which ensures that an irreducible Markov system is globally concentrating. Its proof may be found in $[12$, Lemma 2.4.2] where general Markov operators were considered.

Lemma 2.1. An irreducible Markov system $\left(K_{i(e)}, w_{e}, p_{e}\right)_{e \in E}$ is globally concentrating if there exists a Lyapunov function $L$, bounded on bounded sets, such that

$$
U L(x) \leq a L(x)+b \quad \text { for } x \in K,
$$

where $a, b$ are nonnegative constants and $a<1$.

An irreducible Markov system $\left(K_{i(e)}, w_{e}, p_{e}\right)_{e \in E}$ is called semi-concentrating if for every $\varepsilon>0$ there exist $\alpha>0$ and $\left\{x_{1}, \ldots, x_{s}\right\} \subset K$ such that

$$
\liminf _{n \rightarrow \infty} U^{* n} \mu\left(\bigcup_{j=1}^{s} B\left(x_{j}, \varepsilon\right)\right) \geq \alpha \quad \text { for } \mu \in P(K) .
$$


Lemma 2.2. Suppose that $\left(K_{i(e)}, w_{e}, p_{e}\right)_{e \in E}$ is a contractive Markov system with an average contracting rate $0<a<1$ such that the family $\left\{K_{1}, \ldots, K_{N}\right\}$ is an open partition of $K$. Then $\left(K_{i(e)}, w_{e}, p_{e}\right)_{e \in E}$ is globally concentrating. Moreover for every $\varepsilon>0$ there exists a bounded Borel set $B \subset K$ such that

$$
\liminf _{n \rightarrow \infty} U^{* n} \mu(B) \geq 1-\varepsilon \quad \text { for all } \mu \in P(K) .
$$

Proof. We show that the assumptions of Lemma 2.1 are satisfied. Choose $x_{j} \in K_{j}$ for $j=1, \ldots, N$. Set $L(x)=d\left(x, x_{1}\right)$ for $x \in K$. Then

$$
\begin{aligned}
U L(x) & =\sum_{e \in E} p_{e}(x) d\left(w_{e}(x), x_{1}\right) \\
& \leq \sum_{e \in E} p_{e}(x)\left(d\left(w_{e}(x), w_{e}\left(x_{i(e)}\right)\right)+d\left(w_{e}\left(x_{i(e)}\right), x_{1}\right)\right) \\
& \leq a d\left(x, x_{1}\right)+a \max _{j \in V} d\left(x_{j}, x_{1}\right)+\max _{e \in E, j \in V} d\left(w_{e}\left(x_{j}\right), x_{1}\right) \quad \text { for } x \in K .
\end{aligned}
$$

Hence (2.2) holds with

$$
b=a \max _{j \in V} d\left(x_{j}, x_{1}\right)+\max _{e \in E, j \in V} d\left(w_{e}\left(x_{j}\right), x_{1}\right) .
$$

Now we may formulate the following corollary:

Corollary 2.1. Let $\left(K_{i(e)}, w_{e}, p_{e}\right)_{e \in E}$ satisfy the hypotheses of Lemma 2.2. If $(K, d)$ is a metric space in which sets of finite diameter are relatively compact, then:

(i) the sequence $\left(U^{* k} \mu\right)_{k \geq 1}$ is tight for all $\mu \in P(K)$;

(ii) the Markov system $\left(\bar{K}_{i(e)}, w_{e}, p_{e}\right)_{e \in E}$ has an invariant Borel probability measure $\mu_{0}$;

(iii) the invariant probability measure $\mu_{0}$ is unique if and only if

$$
\lim _{n \rightarrow \infty} \frac{1}{n} \sum_{k=1}^{n} U^{k} g(x)=\int_{K} g d \mu_{0} \quad \text { for all } x \in K \text { and } g \in C(K) ;
$$

(iv) we have

$$
\sum_{j=1}^{N} \int_{K} d\left(x, x_{j}\right) \mu_{0}(d x)<\infty \quad \text { for all } x_{j} \in K_{j}, j=1, \ldots, N .
$$

Proof. (i)-(iii) easily follow from Lemma 2.1 and from Lemma 2.4.2 and Theorem 5.1 in [12]. On the other hand, from the proof of Lemma 2.1 and an induction argument it follows that for fixed $x_{j} \in K_{j}$,

$$
U^{n} L(x) \leq a^{n} L(x)+\frac{b}{1-a},
$$


where $L(x)=d\left(x, x_{j}\right)$ and $n \in \mathbb{N}$. Let $\mu_{0} \in P(K)$ be a unique invariant measure. Then

$$
\begin{aligned}
\int_{K} d\left(x, x_{j}\right) & \mu_{0}(d x)=\limsup _{n \rightarrow \infty} \int_{K} d\left(x, x_{j}\right) U^{* n} \mu_{0}(d x) \\
& =\limsup _{n \rightarrow \infty} \int_{K} U^{n} L(x) \mu_{0}(d x) \leq \limsup _{n \rightarrow \infty} \int_{K}\left[a^{n} L(x)+\frac{b}{1-a}\right] \mu_{0}(d x) \\
\leq & \frac{b}{1-a}<\infty,
\end{aligned}
$$

by the Lebesgue monotone convergence theorem. Hence (iv) follows.

From now on we will assume that $p_{e}, e \in E$, is Dini continuous and let $\Phi_{e}$ be its modulus of uniform continuity. Set $\Phi=\sum_{e \in E} \Phi_{e}$ and observe that

$$
\int_{0}^{c} \frac{\Phi(t)}{t} d t<\infty \quad \text { for every } c>0 .
$$

Then the function $\varphi:[0, \infty) \rightarrow[0, \infty)$ given by

$$
\varphi(t)=t+\sum_{n=0}^{\infty} \Phi\left(a^{n} t\right)
$$

satisfies

$$
\Phi(t)+\varphi(a t) \leq \varphi(t) \quad \text { for } t \geq 0 .
$$

Moreover, we easily check that:

- $\varphi$ is continuous and $\varphi(0)=0$;

- $\varphi$ is nondecreasing and concave;

- $\varphi(t)>0$ for $t>0$ and $\lim _{t \rightarrow \infty} \varphi(t)=\infty$.

We denote by $\mathcal{F}_{\varphi}$ the family of all continuous functions $f: K \rightarrow \mathbb{R}$ such that $|f(x)| \leq 1$ and $|f(x)-f(y)| \leq \varphi(\widetilde{d}(x, y))$ for all $x, y \in K$, where

$$
\widetilde{d}(x, y)= \begin{cases}d(x, y) & \text { if } x, y \in K_{j} \text { for } j \in\{1, \ldots, N\}, \\ \max (c, d(x, y)) & \text { otherwise }\end{cases}
$$

with $c>0$ such that $\varphi(c)>2$. It is obvious that $\widetilde{d}$ is a metric on $K$ equivalent to $d$. The definition of $\widetilde{d}$ looks somewhat sophisticated but it allows us to verify nonexpansiveness merely on one element of the partition of $K$.

Lemma 2.3. Let the assumptions of Theorem 1.1 hold. Then the operator $U$ given by (1.1) satisfies $U\left(\mathcal{F}_{\varphi}\right) \subset \mathcal{F}_{\varphi}$.

Proof. Fix $f \in \mathcal{F}_{\varphi}$. We have

$$
|U f(x)|=\left|\sum_{e \in E} p_{e}(x) f\left(w_{e}(x)\right)\right| \leq \sum_{e \in E} p_{e}(x)=1 \quad \text { for } x \in K .
$$


Further, from (1.3) it follows that

$$
\begin{aligned}
|U f(x)-U f(y)| & =\left|\sum_{e \in E} p_{e}(x) f\left(w_{e}(x)\right)-\sum_{e \in E} p_{e}(y) f\left(w_{e}(y)\right)\right| \\
& \leq \sum_{e \in E}\left|p_{e}(x)-p_{e}(y)\right|+\sum_{e \in E} p_{e}(x)\left|f\left(w_{e}(x)\right)-f\left(w_{e}(y)\right)\right| \\
& \leq \Phi(d(x, y))+\sum_{e \in E} p_{e}(x) \varphi\left(d\left(w_{e}(x), w_{e}(y)\right)\right)
\end{aligned}
$$

for $x, y \in K_{j}, j=1, \ldots, N$. Since $\varphi$ is concave and nondecreasing,

$$
|U f(x)-U f(y)| \leq \Phi(d(x, y))+\varphi(a d(x, y)) \leq \varphi(d(x, y))=\varphi(\widetilde{d}(x, y))
$$

for $x, y \in K_{j}, j=1, \ldots, N$.

If $x$ and $y$ are in different $K_{j}$, then

$$
|U f(x)-U f(y)| \leq 2 \leq \varphi(c) \leq \varphi(\widetilde{d}(x, y)),
$$

which finishes the proof that $U\left(\mathcal{F}_{\varphi}\right) \subset \mathcal{F}_{\varphi}$.

Lemma 2.4. Let the assumptions of Theorem 1.2 hold. Then the irreducible Markov system $\left(K_{i(e)}, w_{e}, p_{e}\right)_{e \in E}$ is semi-concentrating.

Proof. Lemma 2.2 shows that there exists a bounded Borel set $B \subset K$ such that

$$
\liminf _{n \rightarrow \infty} U^{* n} \mu(B)>1 / 2 \quad \text { for all } \mu \in P(K) .
$$

Without loss of generality we may assume that $B_{j}=B \cap K_{j} \neq \emptyset$ for $j=$ $1, \ldots, N$. Fix $\varepsilon>0$. Choose an integer $m \in \mathbb{N}$ such that $a^{m}(\operatorname{diam} B)<\varepsilon$. Further, let $\eta>0$ be such that

$$
(1+\eta)^{m} a^{m} \operatorname{diam} B \leq \varepsilon .
$$

Fix $x_{j} \in B_{j}, j=1, \ldots, N$, and define $C \subset K$ by

$$
C=\bigcup_{j=1}^{N} \bigcup_{e_{1}, \ldots, e_{m} \in E} B\left(w_{e_{m}} \circ \cdots \circ w_{e_{1}}\left(x_{j}\right), \varepsilon\right) .
$$

Now (1.3) implies that for every $y \in K_{j}$ there exists $I_{y} \subset E^{m}$ such that

$$
d\left(w_{e_{m}} \circ \cdots \circ w_{e_{1}}\left(x_{j}\right), w_{e_{m}} \circ \cdots \circ w_{e_{1}}(y)\right) \leq(1+\eta)^{m} a^{m} d\left(x_{j}, y\right)
$$

for $\left(e_{1}, \ldots, e_{m}\right) \in I_{y}$ and

$$
\sum_{\left(e_{1}, \ldots, e_{m}\right) \in I_{y}} p_{e_{1}}(y) p_{e_{2}}\left(w_{e_{1}}(y)\right) \cdots p_{e_{m}}\left(w_{e_{m-1}} \circ \cdots \circ w_{e_{1}}(y)\right) \geq\left(\frac{\eta}{1+\eta}\right)^{m} .
$$

This can be shown by an analysis similar to that in the proof of Lem- 
ma 6.3.1 in [12]. Observe that for every $y \in B_{j}$ and $\left(e_{1}, \ldots, e_{m}\right) \in I_{y}$ we have $w_{e_{m}} \circ \cdots \circ w_{e_{1}}(y) \in C$. Set

$$
\alpha=\frac{1}{2}\left(\frac{\eta}{1+\eta}\right)^{m}
$$

By induction and the definition of $C$ for each $n \in \mathbb{N}$ we obtain

$$
\begin{aligned}
U^{*(m+n)} \mu(C) \geq & \int_{K} \mathbb{1}_{C}\left(w_{e_{m}} \circ \cdots \circ w_{e_{1}}(y)\right) \\
& \times \sum_{\left(e_{1}, \ldots, e_{m}\right) \in I_{y}} p_{e_{1}}(y) \cdots p_{e_{m}}\left(w_{e_{m-1}} \circ \cdots \circ w_{e_{1}}(y)\right) U^{* n} \mu(d y) \\
\geq & \left(\frac{\eta}{1+\eta}\right)^{m} U^{* n} \mu(B) \quad \text { for } \mu \in P(K)
\end{aligned}
$$

and consequently

$$
\liminf _{n \rightarrow \infty} U^{* n} \mu(C) \geq \alpha \quad \text { for } \mu \in P(K),
$$

which finishes the proof.

LEMma 2.5. If an irreducible Markov system is aperiodic, then for every $k, l \in V$ there exist $m \in \mathbb{N}$ and $\left(e_{1}, \ldots, e_{m}\right),\left(\widetilde{e}_{1}, \ldots, \widetilde{e}_{m}\right)$ such that $i\left(e_{1}\right)=k$, $i\left(\widetilde{e}_{1}\right)=l$ and $t\left(e_{m}\right)=t\left(\widetilde{e}_{m}\right)$.

Proof. Fix $k, l \in V$. Let $\left(e_{1}^{k}, \ldots, e_{p}^{k}\right)$ and $\left(e_{1}^{l}, \ldots, e_{q}^{l}\right)$ be paths in $(V, E, i, t)$ starting from $k, l$, respectively and containing all successive cycles from $\mathbf{C}$. Assume that $t\left(e_{p}^{k}\right)=t\left(e_{q}^{l}\right)$. If $p=q$, then the proof is complete. Conversely, assume that $p>q$. Since $\left[l\left(\mathbf{c}_{1}\right), \ldots, l\left(\mathbf{c}_{M}\right)\right]=1$, there exist integers $m_{1}, \ldots, m_{M}$ such that

$$
\sum_{j=1}^{M} m_{j} l\left(\mathbf{c}_{j}\right)=p-q .
$$

Let $J \subset\{1, \ldots, M\}$ be such that $m_{j}<0$ for $j \in J$ and $m_{j} \geq 0$ for $j \in\{1, \ldots, M\} \backslash J$. Adding to $\left(e_{1}^{k}, \ldots, e_{p}^{k}\right)$ the cycle composed of the cycles $\mathbf{c}_{j}$ taken $m_{j}$ times for $j \in J$, and similarly adding to $\left(e_{1}^{l}, \ldots, e_{q}^{l}\right)$ the cycle composed of the cycles $\mathbf{c}_{j}$ taken $m_{j}$ times for $j \in\{1, \ldots, M\} \backslash J$, we finish the proof of the lemma.

\section{Proofs}

Proof of Theorem 1.1. From Lemma 2.3 it follows that the Markov system $\left(K_{i(e)}, w_{e}, p_{e}\right)_{e \in E}$ is semi-concentrating. Thus the statement of Theorem 1.1 follows from Theorem 5.5 in [12] and Lemma 2.1.

Proof of Theorem 1.2. From Theorem 1.1 it follows that $U^{*}$ admits an invariant probability measure. In view of Lemma 2.4, from Theorem 5.4 and Remark 5.1 in [12] it follows that to finish the proof of stability it remains to 
show that for every $\varepsilon>0$ there is $\beta>0$ such that for every $\mu_{1}, \mu_{2} \in P(K)$ there exist a bounded Borel set $A \subset K$ with diam $A \leq \varepsilon$ and $n \in \mathbb{N}$ satisfying

$$
U^{* n} \mu_{j}(A) \geq \beta \quad \text { for } j=1,2 .
$$

Fix $\varepsilon>0$. According to Theorem 1.1 there is a compact set $K_{0} \subset K$ such that

$$
\widetilde{\mu}\left(K_{0}\right) \geq 4 / 5 \quad \text { for all } \widetilde{\mu} \in \bigcup_{\mu \in P(K)} \omega(\mu) .
$$

By the Aleksandrov theorem there exists a sequence $\left(m_{n}\right)_{n \geq 1}$ such that for each open set $G$ with $K_{0} \subset G$,

$$
\liminf _{n \rightarrow \infty} U^{* m_{n}} \mu_{j}(G)>1 / 2 \text { for } j=1,2 .
$$

Consequently, there exist $k, l \in V$ and a subsequence $\left(\widetilde{m}_{n}\right)_{n \geq 1}$ of $\left(m_{n}\right)_{n \geq 1}$ such that

$$
\liminf _{n \rightarrow \infty} U^{* \widetilde{m}_{n}} \mu_{1}\left(G_{1}\right)>\frac{1}{2 N}
$$

and

$$
\liminf _{n \rightarrow \infty} U^{* \widetilde{m}_{n}} \mu_{2}\left(G_{2}\right)>\frac{1}{2 N}
$$

for arbitrary open neighbourhoods $G_{1}, G_{2}$ of $\widetilde{K}_{k}=K_{0} \cap K_{k}, \widetilde{K}_{l}=K_{0} \cap K_{l}$, respectively. By Lemma 2.3 we choose $\widetilde{m} \in \mathbb{N}$ such that for $k, l \in V$ there exist paths $\left(\widehat{e}_{1}, \ldots, \widehat{e}_{m}\right),\left(\widetilde{e}_{1}, \ldots, \widetilde{e}_{m}\right)$ satisfying $i\left(\widehat{e}_{1}\right)=k, i\left(\widetilde{e}_{1}\right)=l, t\left(\widehat{e}_{m}\right)=$ $t\left(\widetilde{e}_{m}\right)$ and $m \leq \widetilde{m}$. Let $F_{k}=w_{\widehat{e}_{m}} \circ \cdots \circ w_{\widehat{e}_{1}}\left(\widetilde{K}_{k}\right)$ and $F_{l}=w_{\widetilde{e}_{m}} \circ \cdots \circ w_{\widetilde{e}_{1}}\left(\widetilde{K}_{l}\right)$. Set $F_{0}=F_{k} \cup F_{l}$ and observe that $F_{0} \subset K_{t\left(\widehat{e}_{m}\right)}$. We easily check that

$$
\liminf _{n \rightarrow \infty} U^{* m_{n}+m} \mu_{j}(\widetilde{G})>\frac{1}{2 N} \delta^{m} \geq \frac{1}{2 N} \delta^{\widetilde{m}} \quad \text { for } j=1,2
$$

and for every open neighbourhood $\widetilde{G}$ of $F_{0}$.

Choose an integer $n \in \mathbb{N}$ such that

$$
a^{n} \operatorname{diam} F_{0} \leq \varepsilon / 3 .
$$

For every path $\left(e_{1}, \ldots, e_{n}\right)$ in the given diagraph define

$$
\pi_{\left(e_{1}, \ldots, e_{n}\right)}(x)=p_{e_{1}}(x) \cdots p_{e_{n}}\left(w_{e_{n-1}} \circ \cdots \circ w_{e_{1}}(x)\right) \quad \text { for } x \in K .
$$

For $x \in F_{0}$ and $\left(e_{1}, \ldots, e_{n}\right)$ such that $i\left(e_{1}\right)=t\left(\widehat{e}_{m}\right)$ we define $O_{\left(e_{1}, \ldots, e_{n}\right)}(x)=\left\{y \in K_{t\left(e_{m}\right)}: d\left(w_{e_{n}} \circ \cdots \circ w_{e_{1}}(x), w_{e_{n}} \circ \cdots \circ w_{e_{1}}(y)\right)<\varepsilon / 3\right\}$. Define

$$
O_{x}=\bigcap O_{\left(e_{1}, \ldots, e_{n}\right)}(x) \quad \text { for } x \in F_{0},
$$

where the intersection is taken over all paths $\left(e_{1}, \ldots, e_{n}\right)$ in the directed graph $\left(K_{i(e)}, w_{e}, p_{e}\right)$ starting from $t\left(e_{m}\right)$. Since $F_{0}$ is a compact set there is 
a finite covering

$$
F_{0} \subset \bigcup_{j=1}^{q} O_{x_{j}}
$$

Set $\widetilde{G}=\bigcup_{j=1}^{q} O_{x_{j}}$. We are going to show that (3.1) holds with $\beta=\delta^{n+\widetilde{m}} /(2 N q)$. Indeed, by (3.4) there exists $M \in \mathbb{N}$ such that

$$
U^{* M+m} \mu_{j}(\widetilde{G})>\frac{1}{2 N} \delta^{\tilde{m}} \quad \text { for } j=1,2 .
$$

Therefore there exist $O_{1}=O_{x_{k}}$ and $O_{2}=O_{x_{l}}$ such that

$$
U^{* M+m} \mu_{j}\left(O_{j}\right)>\frac{1}{2 N} \frac{\delta^{\tilde{m}}}{q} .
$$

By (1.3) and the definition of $O_{1}$ and $O_{2}$ we find a path $\left(e_{1}^{\prime}, \ldots, e_{n}^{\prime}\right)$ such that

$$
A=w_{e_{n}^{\prime}} \circ \cdots \circ w_{e_{1}^{\prime}}\left(O_{1}\right) \cup w_{e_{n}^{\prime}} \circ \cdots \circ w_{e_{1}^{\prime}}\left(O_{2}\right)
$$

satisfies $\operatorname{diam} A<\varepsilon$. Finally,

$$
\begin{aligned}
U^{* M+m+n} & \mu_{j}(A) \\
& =\sum_{\left(e_{1}, \ldots, e_{n}\right)} \int_{K} \mathbb{1}_{A}\left(w_{e_{n}} \circ \cdots \circ w_{e_{1}}(y)\right) \pi_{\left(\widetilde{e}_{1}, \ldots, \widetilde{e}_{n}\right)}(y) U^{* M+m} \mu_{j}(d y) \\
& \geq \delta^{n} \cdot \frac{1}{2 N} \delta^{\widetilde{m}} / q=\frac{\delta^{n+\widetilde{m}}}{2 N q}=\beta \quad \text { for } j=1,2,
\end{aligned}
$$

which finishes the proof.

Acknowledgements. Tomasz Szarek was supported by the State Committee for Scientific Research (Poland) Grant no. 2 P03A 03125.

\section{References}

[1] M. F. Barnsley, S. G. Demko, J. H. Elton and J. S. Geronimo, Invariant measures arising from iterated function systems with place dependent probabilities, Ann. Inst. H. Poincaré 24 (1988), 367-394.

[2] P. Diaconis and D. Freedman, Iterated random functions, SIAM Rev. 41 (1999), $41-76$.

[3] J. H. Elton, An ergodic theorem for iterated maps, Ergodic Theory Dynam. Systems 7 (1987), 481-488.

[4] M. Iosifescu and S. Grigorescu, Dependence with Complete Connections and its Applications, Cambridge Univ. Press, 1990.

[5] A. Lasota and M. C. Mackey, Cell division and stability of cellular populations, J. Math. Biology 38 (1999), 241-261.

[6] A. Lasota and T. Szarek, Dimension of measures invariant with respect to Ważewska partial differential equations, J. Differential Equations 196 (2004), 448-465.

[7] A. Lasota and J. A. Yorke, Lower bound technique for Markov operators and iterated function systems, Random Comput. Dynam. 2 (1994), 41-77. 
[8] R. D. Mauldin and S. C. Williams, Hausdorff dimension in graph directed constructions, Trans. Amer. Math. Soc. 309 (1988), 811-829.

[9] S. Meyn and R. Tweedie, Markov Chains and Stochastic Stability, Springer, 1993.

[10] Ö. Stenflo, Uniqueness of invariant measures for place-dependent random iterations of functions, in: Fractals in Multimedia (Minneapolis, MN, 2001), IMA Vol. Math. Appl. 132, Springer, New York, 2002, 13-32.

[11] T. Szarek, Invariant measures for Markov operators with applications to function systems, Studia Math. 154 (2003), 207-222.

[12] - Invariant measures for nonexpansive Markov operators on Polish spaces, Dissertationes Math. 415 (2003).

[13] I. Werner, Contractive Markov systems, J. London Math. Soc. (2) 71 (2005), 236258.

[14] —, Ergodic theorem for contractive Markov systems, Nonlinearity 17 (2004), 23032313.

Institute of Mathematics

Silesian University

Bankowa 14

40-007 Katowice, Poland

E-mail: horbacz@ux2.math.us.edu.pl

szarek@ux2.math.us.edu.pl

Received November 25, 2005

Revised version November 3, 2006

(5812) 